\title{
Large Calvarial And Brain Metastases Secondary to Papillary Carcinoma of Thyroid
}

\author{
Anamalla Vishnu Vardhan ${ }^{1,}$ Kalyan Bommakanti ${ }^{2}$, \\ Samson sujith kumar Gaddam ${ }^{3}$, Sreevani supula ${ }^{4}$, Kakularam Raghunath Reddy $^{5}$ \\ ${ }^{1}$ (Department Of Neurosurgery, ESI Medical College,Sanath Nagar ,Hyderabad, India). \\ ${ }_{2}^{2}$ (Department of Neurosurgery,Maxcure-Mediciti hospital,Hyderabad, India) \\ ${ }_{3}^{3}$ (Department of Neurosurgery,ESI Medical college,Hyderabad,India) \\ ${ }^{4}$ (Department of Neurosurgery,ESI Medical College,Hyderabad,India) \\ ${ }_{5}^{5}$ (Department of Neurosurgery,ESI Medical college,Hyderabad,India)
}

\begin{abstract}
Cerebral metastases rarely occurs following an episode of papillary carcinoma of thyroid. However, it is proven that inadequate or inadequate treatment of papillary carcinoma of thyroid definitely increases the risk of cerebral and/or distant metastases. In fact, statistics show that nearly 18\% of patients with a history of papillary thyroid carcinoma suffer from distant metastases and the brain is a likely second location for distant spread. This paper reports a case of papillary carcinoma thyroid which was inadequately treated and now presented with cystic cerebral metastases. We also discuss the incidence of papillary carcinoma of thyroid and brain metastases, risks involved, symptoms, treatment options and overall prognosis regarding the condition.
\end{abstract}

Keywords: Brain, Cerbral metastases, papillary carcinoma of thyroid..

\section{Introduction}

Cystic cerebral metastases from a papillary carcinoma of thyroid are rare.This paper discusses one such case where the patient's treatment of his primary thyroid condition was somewhat incomplete and which later resulted in the formation of a large cystic right parieto-occipital lesion. Excision of accessible cerebral metastases was possible followed by routine radiotherapy. The clinical outcome was favorable.

\section{Case Report}

3.1The patient, a 52 year old male, presented with a swelling in the right parieto-occipital region that grew gradually over the past 1 year. It was painless but the swelling increased in size rapidly over the last 1 month. Moreover, since the last 1 month, the patient complained of a rapidly progressing weakness of the left upper and lower limbs. This was complimented by headache accompanied by projectile vomiting during early mornings over the same period and sporadic transient visual loss s/o increased intracranial pressure.

\subsection{Past history:}

The patient already had a history of papillary carcinoma of the thyroid. Thyroidectomy was performed 3 years ago but surgical details were unavailable. No radio-iodine treatment or relevant radiotherapy was given in the post-operative period. The patient did not go for regular follow up with his attending surgeon and came to us with this large swelling in his scalp.

\subsection{Examination findings:}

A large scalp swelling, measuring $20 \mathrm{cms} \times 15 \mathrm{cms}$ in the right parieto-occipital region was noticed. Overlying skin was stretched and shiny. There was no evidence of ulcerations and skin was mobile and there were no signs of inflammation. The swelling had a cystic consistency, was non-compressible, non-pulsatile with no cough impulse. (Fig 1) It was also negative for trans-illumination. Few enlarged lymphnodes were palpable in the cervical region and right axilla. A post-operative scar on the thyroid region was observed. 

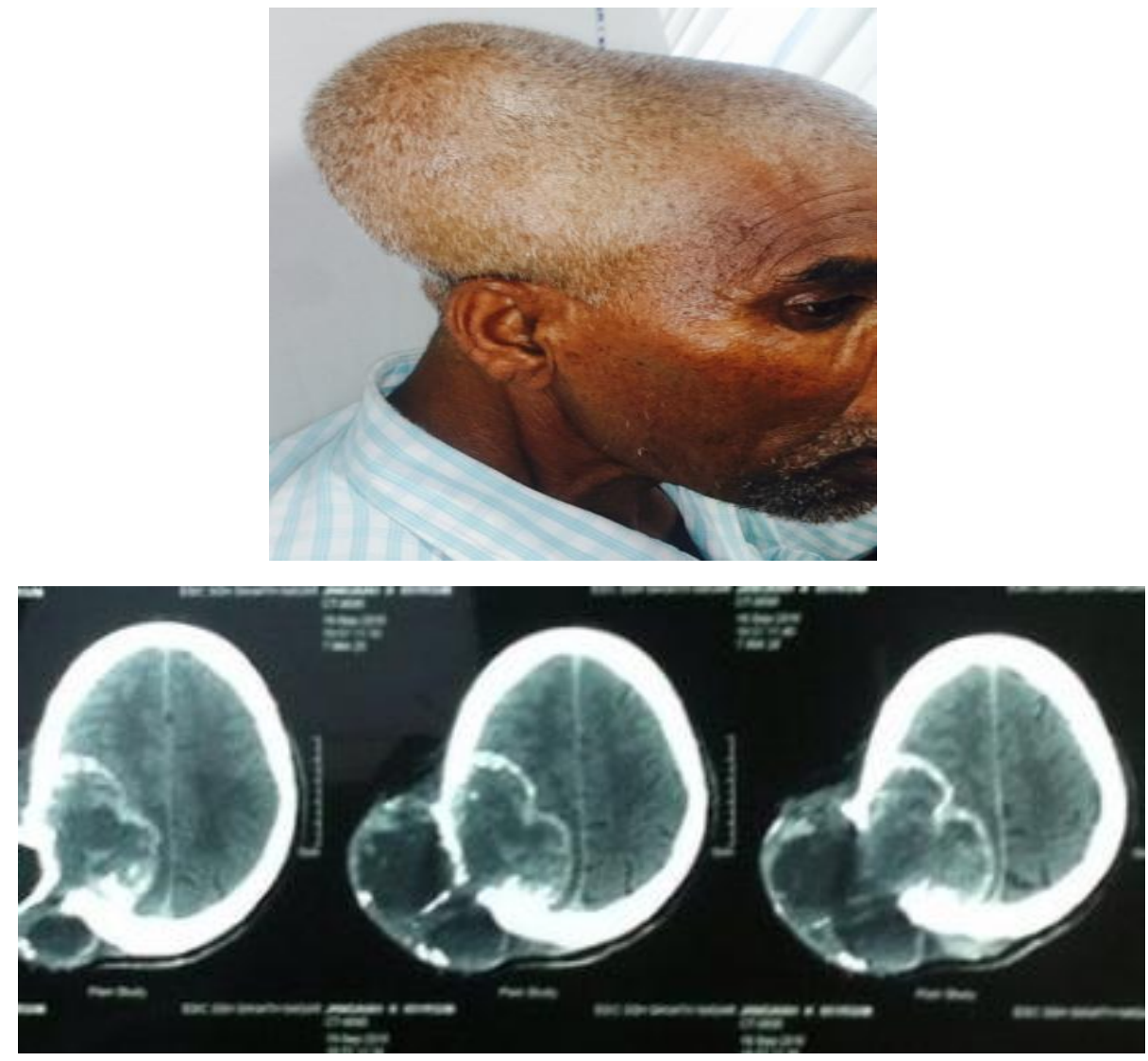

Fig 1: A large scalp swelling, measuring $20 \mathrm{cms} \times 15 \mathrm{cms}$ in the right parieto-occipital region was noticed. Overlying skin was stretched and shiny. There was no evidence of ulcerations and skin was mobile and there were no signs of inflammation

He was fully conscious and coherent. However, left hemiparesis of MRC (Medical Research council) grade 3/5 was present. Examination of fundus revealed papilloedema suggestive of increased intracranial pressure and there was no evidence of spinal tenderness or stiffness in neck. Higher mental functions and other cranial nerves were normal.

\subsection{Investigations:}

Routine investigations including erythrocyte sedimentation rate and C- reactive protein were normal.

1.4.1. CT (Computed Tomography) Brain Plain: Showed a large lesion in right parieto-occipital region. It was solid-cystic in consistency with extracranial and intracranial component. The extracranial component under the skin was cystic with the wall showing small areas of calcification. The underlying bone was eroded due to invasion by the tumor. The intracranial component in the right parieto-occipital region was predominantly cystic with a small hyperdense solid component in the anterior portion. The wall of the intracranial part was also hyperdense with focal areas of further higher density, probably calcifications. There was moderate amount of oedema surrounding the lesion. A second small hyperdense lesion was seen in the left frontal lobe. (Fig 2)

Fig 2: CT brain plain: The extracranial component under the skin was cystic with the wall showing small areas of calcification. The underlying bone was eroded due to invasion by the tumor. The intracranial component in the right parieto-occipital region was predominantly cystic with a small hyperdense solid component in the anterior portion. The wall of the intracranial part was also hyperdense with focal areas of further higher density, probably calcifications. There was moderate amount of oedema surrounding the lesion. A second small hyperdense lesion was seen in the left frontal lobe.

1.4.2 MRI (Magnetic Resonanace Imaging) Brain Plain: Both the intra and extracranial portions were predominantly cystic. The cystic components are hyperintense on T1 weighted and T2 weighted images suggestive of subacute bleed. The cysts were septated. The walls of the lesion and small solid areas are 
hypointense on T1 weighted and T2 weighted images. Contrast enhanced imaging was not done due to financial constraints. Ipsilateral ventricles were compressed and significant midline shift was present. The left frontal lesion was hypointense in T1 weighted and T2 weighted images with moderate surrounding oedema. (Fig 3)

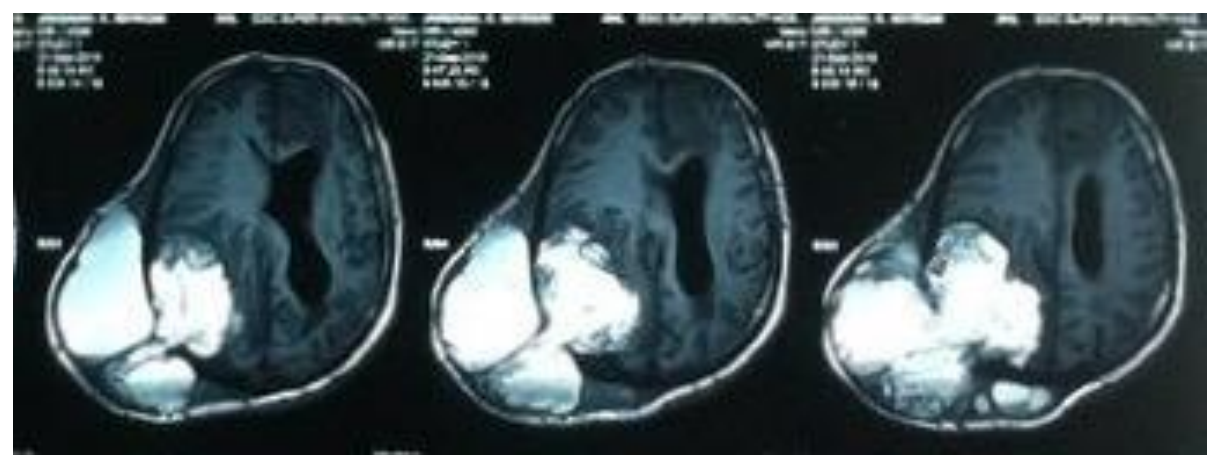

Fig 3: The cystic components are hyperintense on $\mathrm{T} 1$ weighted and $\mathrm{T} 2$ weighted images suggestive of subacute bleed. The cysts were septated. The walls of the lesion and small solid areas are hypointense on T1 weighted and $\mathrm{T} 2$ weighted images

1.4.3 Whole Body PET(Positron Emission Tomography) Imaging: Revealed heterogeneously enhancing lesions with calcifications in both lobes of thyroid with retrosternal extension; Multiple enlarged left supraclavicular, right axillary, anterior mediastinal, prevascular, paraaortic, paratracheal, subcarinal, right hilar, peri-oesophageal and right internal mammillary lymphnodes; Muliple pleural and parenchymal nodular lesions in both lungs; Irregular speculated soft tisse density lesion in right lung lower lobe; Multiple expansile lytic lesions in right parietal bone with large soft tissue component compressing brain parenchyma, right scapula, sternum, multiple ribs, C7, D8, L4, L5 vertebrae, left iliac bone, bilateral acetabulae, left humerus and right femur; Enhancing dural based nodular lesion in left frontal lobe; and Multiple enhancing nodular lesions in right scapula and bilateral thigh muscles.

1.5Treatment: Treatment was based on antiepileptic drugs. Injection dexamethasone $8 \mathrm{mg}$ stat was given, followed by $4 \mathrm{mg}$ thrice a day. Surgery was performed and gross total excision of the right parietooccipital lesion was achieved. The overlying skin was free of the tumor. The tumor was well defined from brain parenchyma and soft with predominantly cystic areas filled with hemorraghic fluid. The small solid components were greyish, moderately vascular and suckable. The wall was thick and well defined. The tumor was eroding the parieto-occipital bone and breaching the dura mater to invade the brain parenchyma. After complete excision,lax watertight duroplasty was performed with pericranial fascia and calvarium was repaired with titanium mesh.

1.6Post-operative Course: Post-operative period was uneventful and the patient was referred to a radiotherapist for further management.

\section{Discusssion}

Although brain metastases from papillary thyroid carcinoma is rare, Dinneen noted that approximately $18 \%$ of patients with distant metastases resulting from papillary thyroid carcinoma developed brain metastases and that the brain was a common second location for distant spread. ${ }^{1}$

4.1 Incidence of papillary carcinoma of thyroid and brain metastases:It is clearly observed that metastatic spread of thyroid carcinoma to the brain is rare. Brain metastases of the cystic nature, occurring from differentiated thyroid carcinoma is extremely rare. Moreover, McConahey of the Mayo Clinic has shown that the presence of extracranial distant metastases is also a risk factor for the development of brain metastases. His studies have shown that nearly $15 \%$ of the patients with metastatic papillary carcinoma developed brain metastasis at some point of time. ${ }^{2}$

4.2Risk factors for brain metastases : Early detection of brain metastasis is crucial because the intracranial metastatic lesion can be life threatening. Statistics show that the reported survival time is usually less than 1 year after brain metastases following thyroid cancer is diagnosed. ${ }^{3,4}$ Appropriate treatment must start immediately to enhance long-term survival because papillary carcinoma of thyroid has a relatively less severe clinical course unless associated with distant metastases. Moreover, given the frequent association of brain metastases coexistent with other sites of distant metastases, it would seem reasonable to consider routine MRI 
examination of the brain in an old patient with thyroid carcinoma who develops any evidence of distant metastatic disease. The presence of brain metastasis is a negative prognostic factor; therefore, treatment must be tailored to the individual patient. Surgery, radiotherapy, and radioiodine treatment have been used with varying results for treatment of brain metastases from thyroid carcinoma. A solitary lesion with complete surgical resection usually results in a better prognosis. ${ }^{3}$

5.3Presenting symptoms: Brain metastasis present with focal neurological deficits like weakness, , cognitive impairment and sensory loss or with symptoms of raised intracranial pressure like headache, vomitings. Therefore, any patient with a history of differentiated thyroid carcinoma with neurological symptoms such as these is a suspect for brain metastasis.

5.4 Treatment options: Treatment for papillary carcinoma of thyroid and brain metastases is not clearly defined and varies on a case to case basis. Patients may be treated conservatively or by surgical resection, external radiation and radioactive iodine. Gamma knife surgery is also an extremely suitable alternative route of treatment, particularly in cases where patients are at a high risk for open surgery or where surgery becomes impossible. Gamma knife radiosurgery was effective in treating isolated lesions with survival rates of up to 61.3 months and worked well in those patients who risked high morbidity in case they underwent craniotomy. ${ }^{5}$ Radiosurgery in fact, is the most effective minimally invasive strategy for brain metastases management. Multiple lesions may be treated through whole brain irradiation and have yielded some very positive results. ${ }^{3}$

Several methods of treatment have been used and the results have mostly been equivocal. However, Chiu observed that surgical resection of brain metastasis helps to prolong survival significantly in such patients. ${ }^{3}$ Moreover, most brain metastases from all cancers happen to be solid and necrosed masses, accompanied by surrounding edema, whereas purely cystic lesions are very uncommon and malignant cells on the walls of these cysts are usually radiotherapy resistant. ${ }^{6}$ Thus, invasive neurosurgical strategies for treatment of the same need to be adopted.

Since thyroid carcinoma is of a slow-growing nature, resection is certainly a right choice of treatment where it is possible or the metastasis is symptomatic. In more aggressive tumors like anaplastic carcinoma for instance, Chiu also reported a significant increase in survival for those patients who underwent surgical resection. Survivals of 18.7 months, 25.5 months and 2.7 months, respectively, were reported in patients undergoing GTR, PR and those without surgery, respectively.

Macroscopic total resection of lesions that are surgically accessible is an excellent treatment method, especially in patients, with a single metastasis or those who are suffering significant mass effect caused by the lesions. If treatment with radioactive iodine is planned, caution should be taken to prevent rapid worsening of tumor growth during thyroid withdrawal, and to minimize any edema that may be the result of treatment. ${ }^{7}$

Chemotherapy, it has been noted, only has a limited role in differentiated thyroid carcinoma management with both extracranial and intracranial metastases. ${ }^{7}$

\subsection{Prognosis}

Papillary carcinoma of thyroid is perhaps the most common form of thyroidal malignancy. However, its prognosis is excellent and distant metastases is also rare. However, should metastases occur in the bones or lungs, the prognosis may be unfavorable. Metastasis of brain from papillary thyroid carcinoma is indeed rare with a frequency of 0.1 to $5 \% .^{5}$

It has also been observed that the prognosis for patient subgroups that develop distant metastasis is unfavorable, particularly in the age groups of $<20$ years and $>60$ years with Grade 3 or 4 cancers and a thyroid lesion of more than $4 \mathrm{~cm}$ as also extra thyroid lesions when initially diagnosed. These groups are more prone to a higher chance of distant metastases and which seriously impacts survival. ${ }^{8}$

\section{Conclusions}

Papillary carcinoma of thyroid rarely causes cerebral metastases; Improper or inadequate treatment of primary thyroid disease increases the risk of cerebral and distant metastases; and excision of accessible cerebral metastases has a favorable clinical outcome.

\section{References}

[1]. Dinneen SF, Valimaki MJ, Bergstralh EJ, Goellner JR, Gorman CA, Hay ID. Distant metastases in papillary thyroid carcinoma: 100 cases observed at one institution during 5 decades. J Clin Endocrinol Metab. 1995;80(7):2041-2045. doi:10.1210/jcem.80.7.7608252.

[2]. Hay ID, McConahey WM, Goellner JR. Managing patients with papillary thyroid carcinoma: insights gained from the Mayo Clinic's experience of treating 2,512 consecutive patients during 1940 through 2000. Trans Am Clin Climatol Assoc. 2002;113:241260. http://www.ncbi.nlm.nih.gov/pubmed/12053713. Accessed January 27, 2017. 
[3]. Chiu AC, Delpassand ES, Sherman SI. Prognosis and treatment of brain metastases in thyroid carcinoma. J Clin Endocrinol Metab. 1997;82(11):3637-3642. doi:10.1210/jcem.82.11.4386

[4]. Biswal BM, Bal CS, Sandhu MS, Padhy AK, Rath GK. Management of intracranial metastases of differentiated carcinoma of thyroid. J Neurooncol. 1994;22(1):77-81. http://www.ncbi.nlm.nih.gov/pubmed/7714554. Accessed January 28, 2017.

[5]. Diyora B, Kamble H, Sharma A, Nayak N. Brain metastasis from papillary carcinoma of thyroid gland. J Neurosci Rural Pract. 2010;1(1):55. doi:10.4103/0976-3147.63109.

[6]. Xu Y-H, Song H-J, Qiu Z-L, Luo Q-Y. Brain metastases with exceptional features from papillary thyroid carcinoma: report of three cases. Hell J Nucl Med. 14(1):56-59. http://www.ncbi.nlm.nih.gov/pubmed/21512667. Accessed January 28, 2017.

[7]. McWilliams RR, Giannini C, Hay ID, Atkinson JL, Stafford SL, Buckner JC. Management of brain metastases from thyroid carcinoma: a study of 16 pathologically confirmed cases over 25 years. Cancer. 2003;98(2):356-362. doi:10.1002/cncr.11488.

[8]. Diyora B, Nayak N, Kamble H, Sharma A. Brain metastasis from papillary carcinoma of thyroid gland. J Neurosci Rural Pract. 2010;1(1):55-57. doi:10.4103/0976-3147.63109. 